\title{
A multimodal mentorship intervention to improve surgical quality in Tanzania's Lake Zone: a convergent, mixed methods assessment
}

\author{
Shehnaz Alidina ${ }^{1 *+} \mathbb{D}$, Leopold Tibyehabwa ${ }^{2 \dagger}$, Sakshie Sanjay Alreja ${ }^{1}$, David Barash ${ }^{3}$, Danta Bien-Aime ${ }^{1}$, \\ Monica Cainer ${ }^{4}$, Kevin Charles ${ }^{4}$, Edwin Ernest ${ }^{2} \mathbb{D}$, Joachim Eyembe ${ }^{5}$, Laura Fitzgerald ${ }^{2}$, Geofrey C. Giiti ${ }^{6} \mathbb{D}_{\text {, }}$ \\ Augustino Hellar ${ }^{2} \mathbb{0}$, Yahaya Hussein ${ }^{7}$, Furaha Kahindo ${ }^{8}$, Benard Kenemo ${ }^{6}$, Albert Kihunrwa ${ }^{6}$, Steve Kisakye ${ }^{9}$,

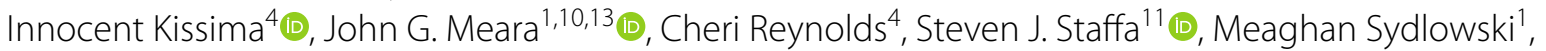

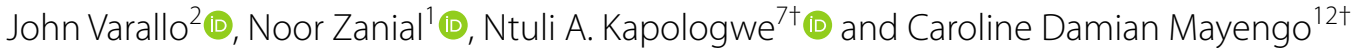

\begin{abstract}
Background: Safe, high-quality surgical care in many African countries is a critical need. Challenges include availability of surgical providers, improving quality of care, and building workforce capacity. Despite growing evidence that mentoring is effective in African healthcare settings, less is known about its role in surgery. We examined a multimodal approach to mentorship as part of a safe surgery intervention (Safe Surgery 2020) to improve surgical quality. Our goal was to distill lessons for policy makers, intervention designers, and practitioners on key elements of a successful surgical mentorship program.

Methods: We used a convergent, mixed-methods design to examine the experiences of mentees, mentors, and facility leaders with mentorship at 10 health facilities in Tanzania's Lake Zone. A multidisciplinary team of mentors worked with surgical providers over 17 months using in-person mentorship, telementoring, and WhatsApp. We conducted surveys, in-depth interviews, and focus groups to capture data in four categories: (1) satisfaction with mentorship; (2) perceived impact; (3) elements of a successful mentoring program; and (4) challenges to implementing mentorship. We analyzed quantitative data using frequency analysis and qualitative data using the constant comparison method. Recurrent and unifying concepts were identified through merging the qualitative and quantitative data.

Results: Overall, $96 \%$ of mentees experienced the intervention as positive, $88 \%$ were satisfied, and $100 \%$ supported continuing the intervention in the future. Mentees, mentors, and facility leaders perceived improvements in surgical practice, the surgical ecosystem, and in reducing postsurgical infections. Several themes related to the intervention's success emerged: (1) the intervention's design, including its multimodality, side-by-side mentorship, and standardization of practices; (2) the mentee-mentor relationship, including a friendly, safe, non-hierarchical, team relationship, as well as mentors' understanding of the local context; and (3) mentorship characteristics, including non-judgmental
\end{abstract}

\footnotetext{
*Correspondence: Shehnaz_Alidina@hms.harvard.edu

†Shehnaz Alidina and Leopold Tibyehabwa are joint first authors

${ }^{\dagger}$ Ntuli A. Kapologwe and Caroline Damian Mayengo are Joint senior

authors

1 Program in Global Surgery and Social Change, Harvard Medical School,

641 Huntington Avenue, Boston, MA 02115, USA

Full list of author information is available at the end of the article
}

(c) The Author(s) 2021. Open Access This article is licensed under a Creative Commons Attribution 4.0 International License, which permits use, sharing, adaptation, distribution and reproduction in any medium or format, as long as you give appropriate credit to the original author(s) and the source, provide a link to the Creative Commons licence, and indicate if changes were made. The images or other third party material in this article are included in the article's Creative Commons licence, unless indicated otherwise in a credit line to the material. If material is not included in the article's Creative Commons licence and your intended use is not permitted by statutory regulation or exceeds the permitted use, you will need to obtain permission directly from the copyright holder. To view a copy of this licence, visit http://creativecommons.org/licenses/by/4.0/. The Creative Commons Public Domain Dedication waiver (http://creativeco mmons.org/publicdomain/zero/1.0/) applies to the data made available in this article, unless otherwise stated in a credit line to the data. 
feedback, experience, and accessibility. Challenges included resistance to change, shortage of providers, mentorship dose, and logistics.

Conclusions: Our study suggests a multimodal mentorship approach is promising in building the capacity of surgical providers. By distilling the experiences of the mentees, mentors, and facility leaders, our lessons provide a foundation for future efforts to establish effective surgical mentorship programs that build provider capacity and ultimately improve surgical quality.

Keywords: Multimodal mentorship intervention, Surgical provider capacity, Surgical quality, Tanzania, Safe Surgery 2020, Workforce

\section{Background}

Recently, the quality and safety of surgical services in African countries has received increasing attention [1-3]. Patients are twice as likely to die after surgery compared to the global average, and postsurgical infection rates are 2 to 10 times higher $[2,4,5]$. Poor surgical quality stems from the convergence of weak health systems, insufficient resources, and workforce challenges. The number of specialists such as surgeons, anesthesiologists, and obstetricians are estimated to be 20-50 times lower in African countries [6] than the recommended minimum needed to provide safe surgical care. (1) While necessary, bolstering the surgical workforce is neither feasible in the short term, nor sufficient on its own [7]. Research in African settings suggests that building the capacity of existing surgical care providers can improve surgical quality [8-11].

The most common method to build healthcare providers' capacity in low-resource settings is training and supervision [12, 13]. However, research suggests that these alone are not sustainable [14] and do not effectively improve performance or quality $[12,15]$. A multipronged strategy incorporating clinical training and practice at the worksite may be more effective [16]. In particular, mentorship, "the process through which an experienced and empathetic person who is proficient in her/his content area teaches and coaches another individual or group of individuals in person and/or virtually to ensure competent workplace performance and provide ongoing professional development" offers a promising strategy for improving surgical provider capacity [17]. Key elements include mutual trust and respect, shared learning objectives, building skills and confidence, and empowerment [18]. Evidence for mentorship's effectiveness in African healthcare settings [19] includes improved infectious disease management [20-22], integrated management of childhood illnesses $[23,24]$, and improved quality of laboratory services $[25,26]$.

Using mentorship to build surgical care provider capacity in low-resource settings is quite recent but $[9,27,28]$ holds promise for three reasons. First, mentorship may be more effective at transferring tacit knowledge and skills following initial training [29] through observation, coaching, and practical solutions in the local context. Second, mentorship may result in more sustainable changes, since it allows surgical providers to continue learning in their environment [30], balance learning with routine work [28], and participate in whole-team learning. Finally, mentorship that provides ongoing learning in smaller amounts spread over time (a "low-dose high frequency approach"), has been effective in adult learning [30,31]; studies have shown that surgical providers in low-resource settings prefer this method [32].

In 2018, a multimodal mentorship component of a Safe Surgery 2020 (SS2020) intervention was implemented to build surgical care provider capacity in 10 health facilities in Tanzania's Lake Zone. SS2020 had two goals: (1) improve surgical quality processes including safety practices, teamwork and communication, and data quality, and (2) reduce postsurgical infections $[8$, 9, 27, 33-35]. Mentorship was the final phase of SS2020 and was designed to support technical and non-technical skill development for non-specialist providers to improve surgical quality, following training [8]. In this paper, we report findings of a mixed-methods assessment of the intervention. By evaluating experiences of mentees, mentors, and facility leaders, our study aims to provide policymakers, intervention designers, and practitioners with information on key elements of a successful surgical mentorship program.

\section{Methods}

\section{Study design}

We used a convergent mixed-methods study design [3638 ] as recommended by experts for the study of complex interventions [39]. We collected quantitative data from surveys and qualitative data from interviews and focus groups. Together, our analyses provided a fuller understanding of the lived experiences of mentees, mentors, and facility leaders with the mentorship intervention [40, 41]. We followed the consolidated reporting guidelines for qualitative research [42]. 


\section{Setting}

Our setting was ten SS2020 intervention facilities in the Mara and Kagera regions in Tanzania's Lake Zone, a focus of national and international efforts, because they are among the most rural and poorest regions [43]. The intervention facilities (health centers, district hospitals, and regional referral hospitals) were selected based on a feasibility assessment conducted by SS2020 partners in 2018 [27]. Non-specialist surgical providers are the backbone of the surgical system, but they work in challenging circumstances: low-provider density and high patient ratios, inadequate surgical infrastructure, and limited training opportunities [44].

\section{Participants}

Our study participants included mentees, mentors, and facility leaders. Mentees were members of the surgical team, including surgical providers, anesthesia providers, theatre nurses, and nurses in postsurgical and postnatal wards. Mentors were a four-person team, including a surgeon or obstetrician/gynecologist, an anesthesia provider, a theatre nurse, and a labor or postoperative ward nurse. Facility leaders included either medical officers-incharge or hospital matrons or patrons.

\section{Selection and preparation}

In the first year, all four mentors came from Bugando Medical Center (BMC), the zonal referral and teaching hospital in the Lake Zone. One year after implementation, for cost and sustainability, two mentors came from $\mathrm{BMC}$ and two from regional facilities. Mentors were selected based on their specialty, clinical experience, and interpersonal skills. BMC mentors participated in leadership and clinical trainings with mentees and received 3 days of training on mentorship. Regional mentors received 3 days of training on mentorship and worked side-by-side with BMC mentors for 6 months.

\section{Multimodal delivery}

A multimodal approach to mentorship (Table 1) was implemented starting June 2018. For in-person mentorship, the mentor team visited each facility bi-monthly for 2 days. Mentors debriefed with surgical teams on their progress with their surgical quality improvement plan-developed after leadership training-and any perioperative and organizational challenges. Next, each mentor focused on one of three tracks: (1) the clinical track included side-by-side coaching in the operating room (OR) on surgical skills, safety practices, and teamwork and communication. Mentors also participated in ward rounds and provided hands-on coaching on postoperative care; (2) the data track included reviewing patient files for completeness of documentation and quality of care and coaching on monitoring and use of data to improve surgical quality; and (3) the patient pathway track examined care from the facility gate to discharge. Mentors debriefed with the surgical team, facility leaders, and Council Health Management Teams, problem-solved, and developed an action plan identifying opportunities and strategies for improvement.

Telementoring was provided through the Project ECHO (Extension for Community Healthcare Outcomes) platform using a hub and spoke approach; mentors (the hub) partnered with surgical teams (the spokes) to facilitate sharing of knowledge and expertise through videoconferencing [45-49]. Biweekly 80-min telementoring sessions were open to all relevant staff. Mentors shared knowledge on a variety of topics through didactic presentations. Next, surgical teams presented cases on best practices or a challenging case related to that topic, followed by peer-to-peer and mentor feedback. Regional WhatsApp groups were established to facilitate further communication, sharing of knowledge and monthly surgical data, and real-time problem solving.

\section{Data collection and analysis Data collection}

Members of the research team, (SA, MS) with experience in global health and implementation science developed a survey based on the goals of the intervention and from literature on mentorship in African healthcare settings [19]. The 57 items focused on six topics: (1) satisfaction with mentorship; (2) perceived impact; (3) experience with mentorship; (4) important mentor characteristics; (5) challenges to implementing mentorship; and (6) respondent characteristics (Additional file 1). The survey was tested in five health facilities in a neighboring region with surgical providers. A paper-based survey was administered 14 months after the start of the intervention to mentees who were interviewed. No survey identifiers were collected, and no incentives were offered for completion.

Two research assistants (NZ, MS) experienced in qualitative research with master's degrees in global health conducted in-depth, semi-structured interviews with mentees, mentors, and facility leaders 14 months after initiation of the intervention. To recruit mentees and facility leaders, we identified the necessary stakeholders (one facility leader and two to three members of the surgical team); the facility or surgical team leaders identified the interviewees. Interviews were $30 \mathrm{~min}$ and conducted in a private space by a research team member; daily field notes were recorded after. Three protocols were used for mentees, mentors, and facility leaders and included questions about perceived impact, the multimodal approach, 


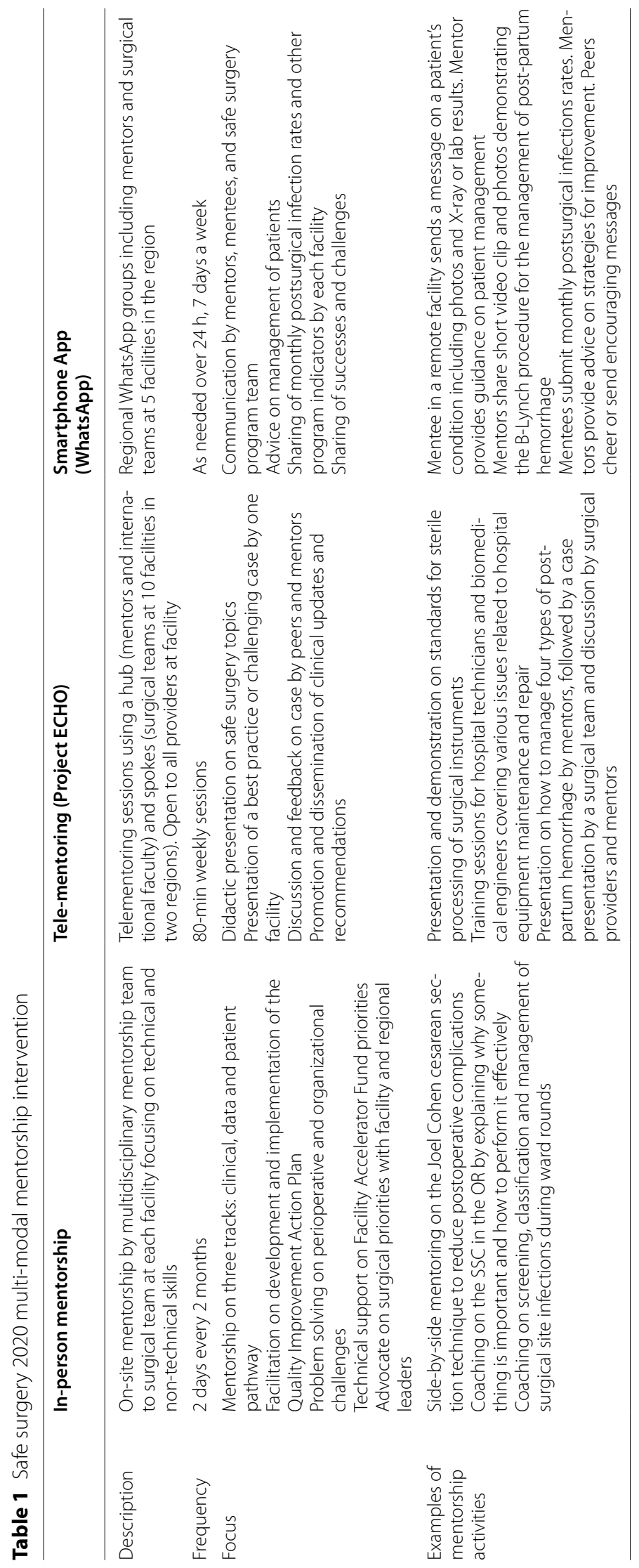


which areas of mentorship were most valued, menteementor relationships, and challenges (Additional file 2). Interviews were conducted in English with translation to Kiswahili when required by Tanzanian SS2020 staff, audiotaped and transcribed. No interviewee ended the interview early or declined to participate. Transcripts were checked for accuracy and uploaded to NVivo V.11 (QSR International, Melbourne, Australia) for coding.

A research assistant (MS) conducted one focus group per facility 10 months after telementoring, with daily field notes. Focus groups included a range from 4 to 15 interviewees per site (facility leaders and members of the surgical team) and lasted $30 \mathrm{~min}$. Questions covered perceived impact, most useful areas of mentoring support, what was best delivered through a virtual platform, challenges, and sustainability (Additional file 2).

\section{Data analysis}

We used descriptive statistics to summarize the percentage of responses on the Likert scale for each survey question. Denominators included the actual number of responses to the question. Statistical testing was not conducted as this was an exploratory study and the sample size was small $(n=25)$. All analyses were performed using Stata version 16.0, (StataCorp LLC. College Station, TX).

Qualitative data were analyzed by three researchers (NZ, DBA, SA) using the constant comparative method to understand the elements of a successful mentorship program $[41,50]$. Researchers reviewed three transcripts to arrive at a preliminary codebook and tested it against nine new transcripts. As new data were coded, we compared the new text segments to those previously assigned the same code; codes were refined to ensure validity until no new codes emerged (i.e., theoretical saturation) [51, 52]. Development of the coding structure, coding definitions, and principles used in applying the codes were documented. Inter-rater reliability between two coders was high $(\kappa=0.87)$ [53], and all 45 transcripts were analyzed using the final codebook. Recurrent and unifying concepts were identified, and the qualitative and quantitative data were merged to identify the major patterns and themes.

\section{Results}

The ten facilities included regional hospitals, district hospitals, and health centers (Table 2). The typical facility was a 101-300 bed government-operated facility performing an average of 90 major surgeries per month. Our response rate for the survey was $82 \%$. Interviewees $(n=45)$ included mentees $(62.2 \%)$, mentors $(17.8 \%)$, and facility leaders $(20 \%)$.
Table 2 Characteristics of intervention facilities and participants, 2019

\begin{tabular}{|c|c|}
\hline \multicolumn{2}{|l|}{ Facility characteristics $(N=10) n(\%)$} \\
\hline \multicolumn{2}{|l|}{ Level of facility } \\
\hline Health Centers & $2(20)$ \\
\hline District Hospitals & $6(60)$ \\
\hline Regional Referral Hospitals & $2(20)$ \\
\hline \multicolumn{2}{|l|}{ Geography } \\
\hline Rural & $5(50)$ \\
\hline Urban & $3(30)$ \\
\hline Suburban & $2(20)$ \\
\hline \multicolumn{2}{|l|}{ Number of inpatient beds } \\
\hline $0-100$ & $3(30)$ \\
\hline $101-300$ & $6(60)$ \\
\hline $300+$ & $1(10)$ \\
\hline Average monthly major surgeries per facility & 90 \\
\hline \multicolumn{2}{|l|}{ Average number of surgical providers per facility } \\
\hline Surgeons & 0.2 \\
\hline Obstetricians/gynecologists & 0 \\
\hline Anesthesiologists & 0 \\
\hline Medical Officers performing surgery & 4.1 \\
\hline Assistant Medical Officers performing surgery & 3.7 \\
\hline Non-physicians proving anaesthesia & 2.8 \\
\hline \multicolumn{2}{|l|}{ Participant characteristics } \\
\hline \multicolumn{2}{|l|}{ Survey $(N=25) n(\%)$} \\
\hline \multicolumn{2}{|l|}{ Role } \\
\hline Surgical provider & $11(39.1 \%)$ \\
\hline Anaesthesia providers & $5(21.7 \%)$ \\
\hline Nurse & $7(30.4 \%)$ \\
\hline Other (facility leader also a surgical provider) & $2(8.7 \%)$ \\
\hline \multicolumn{2}{|l|}{ Years in role } \\
\hline$<1$ year & $1(4.3 \%)$ \\
\hline $1-3$ years & $7(30.4 \%)$ \\
\hline $3+$ years & $14(60.9 \%)$ \\
\hline Missing & $1(4.3 \%)$ \\
\hline \multicolumn{2}{|l|}{ Present for mentorship visits } \\
\hline$<3$ & $3(13 \%)$ \\
\hline $3+$ & $18(78.3 \%)$ \\
\hline Missing & $2(8.7 \%)$ \\
\hline \multicolumn{2}{|l|}{ Interviews (N=45) n (\%) } \\
\hline \multicolumn{2}{|l|}{ Role } \\
\hline \multicolumn{2}{|l|}{ Mentees } \\
\hline Surgical provider & $12(26.7 \%)$ \\
\hline Anaesthesia provider & $5(11.1 \%)$ \\
\hline Nurses & $11(24.4 \%)$ \\
\hline Facility leader & $9(20 \%)$ \\
\hline Mentor & $8(17.8 \%)$ \\
\hline \multicolumn{2}{|l|}{ Focus Groups $(N=10)$} \\
\hline $\begin{array}{l}\text { Attendees per focus group (surgical providers, anesthesia } \\
\text { providers, facility leaders) }\end{array}$ & $4-15$ \\
\hline
\end{tabular}


Below we present quantitative and qualitative results for our study, including perceptions related to the successful impact of mentoring, three themes, and eleven constituent subthemes that contributed to the successful impact and four challenges to implementing mentorship. Table 3 provides illustrative quotations for themes that emerged from the interviews. Quotations have been edited for conciseness.

\section{Perceived mentorship impact}

Overall, $96 \%$ of mentees experienced the intervention as positive, $88 \%$ were satisfied, and $100 \%$ supported continuing the intervention in the future (Additional file 3). All mentees reported they had changed their surgical practices due to mentorship, and $86 \%$ of mentees said their facilities made positive changes (Additional file 4). One leader suggested that, in time, they could become mentor hospitals to other facilities.

Three quarters of all mentees reported mentorship had made a difference "to a great extent" in five areas: confidence, Safe Surgical Checklist (SSC) use, asking for case consultations, teamwork and communication, and clinical skills (Fig. 1). The qualitative data supported these positive survey findings. All participants perceived that mentorship helped mentees improve their confidence, surgical skills, safety practices, teamwork and communication, and safety culture.

Mentors also reported their own clinical skills, surgical practices, and teamwork and communication skills improved from participating in the SS2020 trainings and mentoring. In addition, they reported their leadership skills and confidence had increased.

All participants perceived improvements in the surgical ecosystem, such as better preoperative and postoperative care. In interviews, leaders and surgical providers mentioned increased revenue because of reduced referrals out, increased surgical volume, and improved best practices. Mentees reported improvements had a positive impact on patients, including reduced postsurgical infections, increased safety, improved communication, faster recovery, and reduced decision-to-incision times.

Below we present the themes and subthemes that contributed to the positive impact.

\section{Valuable elements of the mentorship intervention Multimodality of the mentorship intervention}

In interviews, participants reported that the all three platforms-in-person mentorship, telementoring, and WhatsApp (Table 1)-were valuable, because they complemented and reinforced each other, contributing to continuous and deeper learning.

Participants explained that in-person mentorship was optimal for learning tacit knowledge and skills such as how to perform certain surgical techniques. In-person mentorship included side-by-side coaching allowing mentors to role-model important technical and nontechnical skills to mentees; a few participants expressed this way of learning was particularly suited to their culture and allowed mentoring to be tailored to the facility's individual context. Mentors said it allowed them to assess the team environment or the quality of their OR registers, follow-up on progress, problem-solve organizational or perioperative issues, and advocate for surgical priorities with facility, district, or regional management. Mentees reported that they valued the real-time communication with mentors to share experiences. There were two challenges of in-person mentorship: (1) it was resource intensive, and (2) a heavy caseload or the lack of a specific type of case on the day of mentoring may make it difficult to engage in mentorship activities.

Participants noted that telementoring was optimal for learning explicit knowledge, and it expanded their knowledge on safe surgery. It was perceived as time-efficient and less costly, since many people could learn together and mentors did not have to travel. It was also a valuable platform for learning about uncommon cases and convenient to bring in different experts. It was open to everyone which helped to create a learning community on safe surgery and promoted shared learning between surgical teams at different facilities with common contexts and challenges. Logistical challenges included after-work timing of sessions, poor internet connectivity, and lack of translation for English sessions by international faculty. Staff workload prevented some mentees from attending.

WhatsApp was a valuable platform for timely advice on patient management and coordination issues regardless of location. Participants highlighted its convenience, since texts or videos could be reviewed at any time. It also created a sense of community between the surgical teams at various facilities. However, some mentioned issues with confidentiality of information and inaccessibility to WhatsApp as disadvantages.

\section{Supportive side-by-side clinical coaching}

Mentees prioritized clinical skills highest among all areas of mentorship received. The top three areas mentees prioritized as "greatly important" were also the areas, where they received mentorship "to a great extent": clinical skills (95.7\% said it was "greatly important" and 76.2\% said they received it "to a great extent"), SSC implementation $(87 \%$ and $66.7 \%$, respectively), and utilization of data $(78.3 \%$ and 59\%, respectively) (Additional file 5).

In interviews, mentees prioritized side-by-side clinical mentorship, because it provided practical, efficient, and direct teaching and feedback with tangible improvements, but there were some differences by discipline. 
Table 3 Illustrative quotations of themes about features of and challenges to a successful mentorship intervention

\section{Themes and sub-themes}

Valuable elements of the mentorship intervention

Multimodality of the mentorship intervention

Supportive side-by-side clinical coaching

Standardization of practices

Useful features of the mentor-mentee relationship Relationship-building

Friendliness

Psychological safety

Mentors as part of the surgical team

Understanding of context

Helpful characteristics of the mentor

Non-judgmental feedback

Mentor experience level

Accessibility of the mentor

Challenges to mentorship

Resistance/lack of buy-in

Shortage of surgical providers

Mentorship dose

Logistical challenges

\section{Illustrative Quotations*}

They are all valuable, because they all depend on each other and none can stand on behalf of the other. (Surgical Provider, Region 1, Facility 2)

They will perform the first case and we observe and identify the gaps that they have. The second case we all scrub in together -both the mentors and the mentees - and we can perform together so in that way we can impact the knowledge through doing procedures together. (Mentor 1)

Nowadays we hardly forget the use of the checklist. The theater staff now has a system of preparing the trays for vaginal cleansing prior whereas in the past days that was not present. Therefore, when there is a ruptured membrane you could just order for a tray that has been prepared and sterilized so it has changed the way people work. (Region 1, Facility 3, Facility Leader)

If you have a mentorship relationship it has to be close. Even if you have something it can be easily shared with them. This makes it easy even to share knowledge and interest between mentors and mentees and it can increase the relationship, because you know each other even outside work and if you have something to ask you may communicate with him or her. (Region 2, Facility 4, Surgical Provider)

We get feedback from mentees and most of the time, they give us feedback that they benefitted from what we offered them and the way we offered is friendly... and we participated as part of their team. And all this is because we had training before we performed mentorship compared to the formal medical training in Tanzania where there is a gap between a lecturer and a student. (Mentor 5)

It is a fine one, because someone who is not a dictator to you, you may have a conversation and you are able to exchange views. We are comfortable to admit mistake and ask questions and help. (Region 2, Facility 4, Surgical Provider)

The relationship is good, because when they come here we work together and they become like team members of the facility. We work together like team members for quality improvement of services. (Nurse, Region 1, Facility 4)

Mentors were trying to understand the local context and condition of the facility, and we started from the entrance gate. (Region 2, Facility 3, Nurse)

The mentors were not judgmental as in once you fail they will not judge you. So it is a conversation. They tell you something and you will ask questions and they will correct like'do this and you were not supposed to do this' in a guiding manner and they give a chance to ask questions. (Surgical Provider, Region 2, Facility 4)

I think when you bring mentors at the facility, they should be senior mentors. For example, I am a senior nurse anaesthetist. When you bring a mentor that is junior to me like someone who has been practicing for less than 6 months in the field then usually cannot add value to me. (Anaesthetist, Region 2, Facility 1) The mentors are skilled, the whole team is skilled from the surgeon, anesthetist and the nurse are all skilled so when they come and they face the challenges of the facility they can assist and tackle together with discussion. (Region 1, Facility 1, Surgical Provider)

They leave behind their numbers and they let people know that they are available so when someone is in trouble they can contact them... There was a time they [surgical team] had a fistula patient, they communicated, and they found a way forward so it has been a team. (Facility Leader, Region 2, Facility 2)

The challenge is some of the providers were taking this program like it belonged to those that only attended the [SS2020] training. They were not ready to involve directly on the mentorship program so sometimes you may find that when mentors come to the facility there is high effort used to get all members that are needed for the program. There is still some resistance at the facility in relation to the mentorship program. (Surgical Provider, Region 1, Facility 2)

Some of the barriers are time, because you may find people have other activities to attend to during the time of presentations/sessions... Another thing is the shortage of staff. Providers may be alone in the ward and it is difficult for them to leave patients and attend the sessions. (Focus Group Discussion, Region 1, Facility 2)

I think the frequency can increase - at least that they should come monthly and they could stay at least if possible for a week for the mentorship. (Region 2, Facility 1, Surgical Provider)

The main problem in this is the language barrier, because our country is based very much on Swahili. . It would be better to have handouts and translation if possible in Swahili. Sometimes the internet is a problem and it is not stable. (Focus Group Discussion, Facility 1, Region 1) 


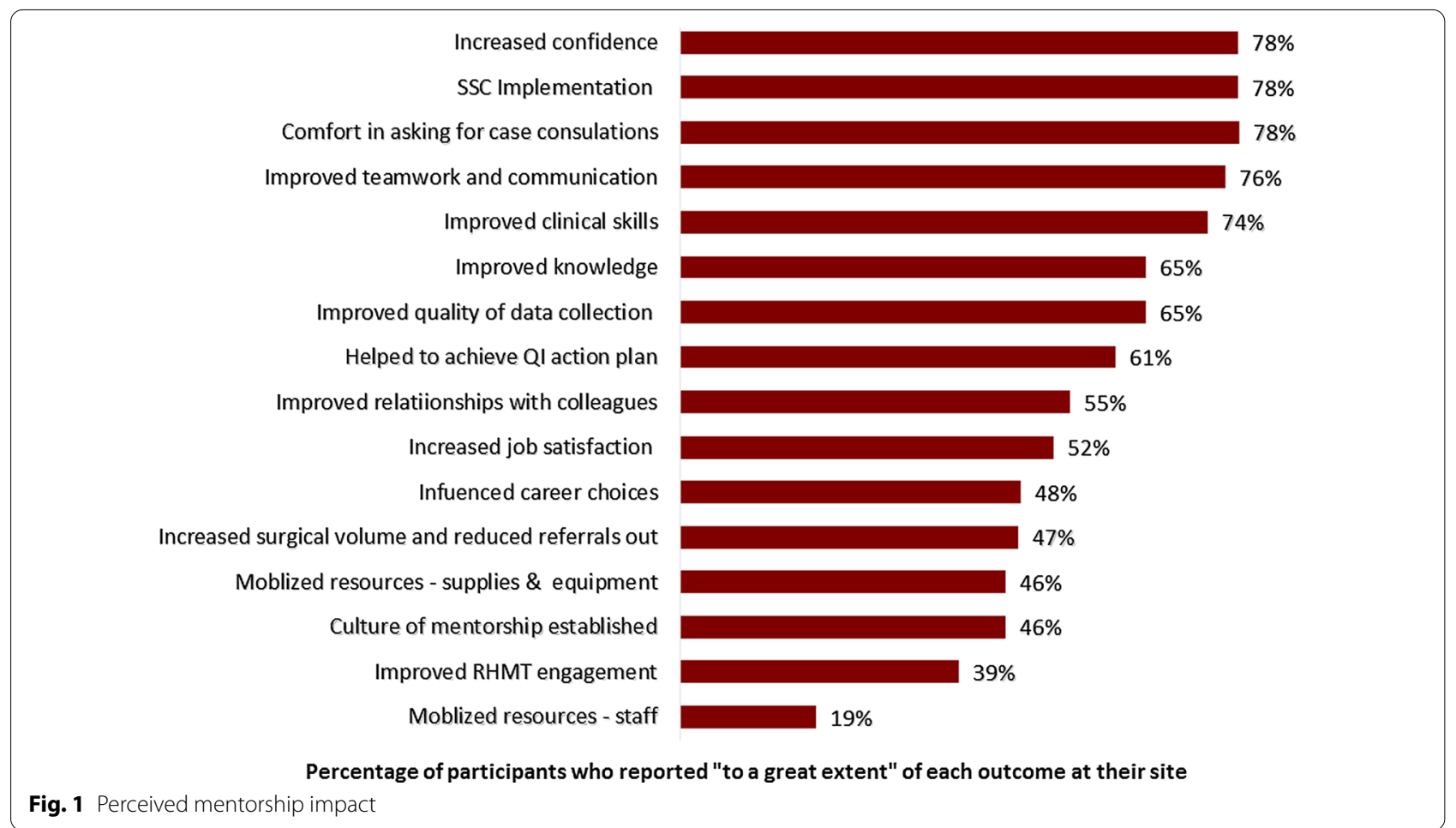

Surgical providers, nurses, and leaders valued mentorship on clinical procedures the most, followed by the SSC, while anesthetists valued the SSC the most, followed by the patient pathway.

\section{Standardization of surgical practices}

Mentors noted that previously, surgical providers and mentors had their own surgical practices at their facilities; after the SS2020 trainings and mentorship, evidencebased practices became standardized. They perceived a culture of following standards improved the quality of care for patients and reduced postsurgical infections.

\section{Helpful features of the mentee-mentor relationships Relationship building}

Mentees and mentors discussed how the mentors first invested in building relationships. Mentees felt that this approach opened the door for mentors to share their knowledge and skills, making it easier for mentees to ask questions and be receptive to feedback for improvement.

\section{Friendliness}

The term "friendly" was most often used by mentees to describe their relationship with mentors due to their non-hierarchical, collegial approach. Furthermore, they felt that the approach to feedback, where mentors started with positive feedback first before discussing areas for improvement reduced resistance. One mentor attributed their ability to build a friendly relationship with mentees to the leadership training they attended together.

\section{Psychological safety}

Mentees described their relationship with their mentors as psychologically safe, where they felt comfortable asking questions, seeking help, and admitting mistakes. Mentors said they were also comfortable discussing feedback with mentees. Mentees and mentors said they shared feedback with each other at the end of each visit, through annual mentor evaluations, and at an annual mentorship debriefing session.

\section{Mentors as part of the surgical team}

Importantly, mentees and facility leaders described their mentors as part of a team working together in the pursuit of surgical quality improvement. One facility leader explained that by becoming part of the team, mentors overcame the common challenge of getting people engaged and it was the sense of being one team that made mentoring a success.

\section{Understanding of local context}

Mentees and leaders felt that mentors understood their context, such as resources, staffing, and culture, and were able to help with their challenges. They noted that 
the patient pathway track, where mentors and mentees reviewed services from the gate to discharge provided mentors with a deeper understanding of their facility. This understanding of context allowed mentors to relate to mentees; as a result, knowledge and skills were transferred more easily.

\section{Useful mentor characteristics}

The top three mentor characteristics mentees prioritized as "greatly important" and exhibited "to a great extent" were interpersonal skills $(90.4 \%$ felt these were "greatly important" and $81 \%$ felt mentors exhibited them "to a great extent"), role modeling (82.6\% and $65.2 \%$, respectively), and courtesy and respect $(81 \%$ and $81 \%$, respectively) (Additional file 6). In interviews, participants raised the importance of mentors who provided nonjudgmental feedback, had sufficient experience, and were accessible.

\section{Non-judgmental feedback}

Mentees highly valued the mentors' nonjudgmental feedback. Mentees mentioned mentors fostered a supportive environment by providing feedback, offered guidance and a chance to ask questions with the goal of learning and improvement.

\section{Mentor experience level}

When describing attributes, many mentees spoke very positively about the experience level of mentors. However, some felt that their mentors lacked sufficient experience in their discipline or in mentorship and were not able to be as helpful as a result.

\section{Accessibility of the mentor}

Mentees discussed they could reach out to their mentors outside mentorship visits if they had a difficult surgical case, which was very helpful. One leader provided an example of when the surgical team reached out to their mentors for advice on the care of a patient with a fistula and worked together as a team.

\section{Challenges to mentorship}

Four themes emerged as barriers to successful implementation of mentorship. First, while most mentees had a good attitude towards mentorship and welcomed it, participants mentioned there was resistance from some mentees. Reasons mentioned included: First, mentees initially perceiving it as another supervision program, less buy-in from mentees who had not attended the SS2020 trainings, and difficulty changing practice. Second, most facilities faced a shortage of surgical providers, so mentees were not prepared when mentors arrived or needed to attend to routine duties during mentorship; afterhours sessions were also unpaid. Third, while some mentees felt the mentorship 'dose' was just right, others felt the frequency and duration of mentorship was insufficient. Finally, there were logistical challenges including communication around timing of the mentorship visits, after work hours of telementoring sessions, poor internet connectivity, and language barriers with international faculty.

\section{Discussion}

We examined a multimodal approach to mentorship as part of a safe surgery intervention to improve surgical quality in Tanzania's Lake Zone to distill lessons on its key elements of success (Box 4).

Our results suggest that mentorship can act as a vehicle to build capacity of surgical providers in low-resource settings. Mentees, mentors, and facility leaders perceived improvements in surgical practice and teamwork in the OR, strengthening of the surgical ecosystem, and a reduction in postsurgical infections; this is supported by our quantitative findings [8]. We attribute the success of the intervention to its multimodal design, collaborative relationships between mentees and mentors, and its grounding in local context.

Most studies examining mentorship in surgery are based in high-income countries [29]. While research on surgery in low-resource settings is sparse [19, 28, 54], our findings are consistent with the emerging evidence about elements of successful mentorship. Mentorship that follows training, uses a side-by-side approach, has high-quality mentee-mentor relationships, understands the local context, and provides nonjudgmental feedback leads to success in low-resource settings [17-19, 24, 55].

A key feature of the intervention was its multimodality. Our goal was to improve surgical quality, which required the development of technical and non-technical skills, behavior changes, and new ways of thinking. A multimodal mentorship approach offered a flexible journey to transform surgical practice and culture. In-person mentorship was optimal for transferring tacit knowledge and skills, telementoring for explicit knowledge, and WhatsApp for real-time problem-solving. This approach allowed for a balance of work and learning, attention to contextual factors, and integration of knowledge and practice. Our findings contribute to the evidence on the effectiveness of multimodal mentorship approaches [56-60].

Relationship building between mentees and mentors was also important for success. To build relationships, mentors presented themselves to mentees as a helper, not as an instructor or a manager. They created a safe, nonjudgmental environment, where mentees felt comfortable sharing their challenges and were receptive to feedback. 
Box 4 Lessons for implementing a successful mentorship intervention to strengthen surgical services in low-resource settings

Intervention design

- A multimodal mentorship intervention design using both in-person and virtual platforms can support different types of learning (e.g., tacit or explicit). The different platforms complement and reinforce each other, contributing to continuous and deeper learning

- Mentorship is optimized when it is part of a multicomponent intervention. Training mentees and mentors on evidence-based practices before mentorship ensures that everyone is working to implement the same standards for safe surgery

- A team-based approach to mentorship can provide discipline-specific mentorship (e.g., nurses mentoring other nurses), and reinforce a culture of shared learning

- To improve the intervention, there should be opportunities for reflection and learning. Incorporating time for bi-directional feedback, such as debriefing after each visit, at annual meetings, and evaluations can strengthen future intervention design

Mentors

- Selecting the right mentors is key. Subject matter expertise and strong interpersonal and communication skills are crucial. Selecting local mentors can facilitate cultural congruence and an understanding of context, relatability, and language. Local mentors can also train new surgical providers more frequently and engage in peer-to-peer learning to diffuse knowledge quickly and continuously

- Preparation of mentors should cover subject matter expertise, change management skills, and mentorship skills, such as relationship-building, communication and feedback, and effective teaching. Pairing junior mentors with experienced mentors can also be considered to strengthen mentorship skills and confidence

- Mentorship requires resources. Mentors need protected time away from clinical work to prepare and conduct mentorship visits as well as resources for coordination, training, and support. Options for incentivizing mentors through compensation, continuing education credits or other incentives like certification should be considered

Implementation

- A Quality Improvement Action Plan can facilitate a shared understanding about the overall improvement goals of the intervention. An action plan can lay out a clear strategy (e.g., specific actions, responsibilities, timing and means of verification) and can provide a framework for assessing progress and setting goals for the next visit

- Buy-in from the surgical team is essential before starting the mentorship intervention; they must understand the goals. It is especially important to address those who are less ready to change. Whole-site orientation and training and engaging facility leaders in mentorship can increase buy-in

- Leadership support and engagement from facilities, district and regional leaders is necessary for success. Leaders can support staff in implementing mentoring activities, release staff time, and assist in setting up QI systems. Furthermore, leadership support is crucial in sustaining surgical quality improvement. Mentorship cannot work if leadership is not receptive to it

- Time constraints must be considered for mentees and mentors. Health facilities in low-resource settings are often faced with staff shortages. Mentors also have competing work and personal demands. Therefore, implementation must consider providing surgical providers the time to learn and improve, timing of sessions, lowering work burden, and revamping tools for efficiency for both mentees and mentors

Sustainability

- Building a culture of mentorship is necessary for sustainability. Mentorship is a promising approach for scaling surgical quality and requires policy support to institutionalize it. Mentorship should be incorporated in the safe surgery space, linked to continuing professional development systems, and should be incorporated in the District plans and budget. Training a pool of local multidisciplinary mentors is critical for cost effectiveness and sustainability

Mentors from regional and local hospitals understood the local context and were perceived as colleagues. A team-based approach which provides discipline-specific mentorship (e.g., nurses mentoring other nurses), can also facilitate technical congruence [18]. Properly training mentors was crucial; during the COVID-19 pandemic mentorship became fully virtual, which required a different skill set than in-person mentoring. Preparation covered how to structure sessions, develop activities, and effectively facilitate virtual sessions.

Literature points to the importance of bridging the knowing-doing gap [34, 61-63]. While most mentees had a positive attitude towards mentoring, there was some resistance from others. It is important to cultivate buy-in by orienting the surgical team to the goals of mentorship, inviting their input on priorities, and training the whole team. Leadership engagement is also critical for successful and sustainable mentorship. Leaders can signal its priority, release staff time for mentorship activities, and create structures that build learning capacity [33, 64-67].

Sustainability requires policy support and a culture of mentorship. It is necessary to engage with national health system leaders to develop policy that integrates surgical mentorship into the existing health system and incorporates it in district plans and budget, including training and compensation for mentors. The concept of mentorship should be introduced during surgical training, incorporated into mentors' regular responsibilities, and linked to continuing professional development [17].

Future research should consider experimental and longitudinal designs to identify which features of the mentorship program are associated with significant improvements in surgical quality. In addition, studies should examine the cost-effectiveness of multimodal mentorship approaches in comparison to unimodal 
approaches. Research should also consider what contextual factors are important for successful surgical mentorship in African settings.

Our study has limitations. Our findings should be confirmed in diverse contexts to generalize results. Our survey did not undergo formal testing to establish reliability and validity. Data were self-reported, so the findings may appear more positive due to social desirability bias [68], and language barriers may have limited the discussion. The qualitative data from interviews may be confounded by participant experiences with other SS2020 intervention components. A key strength of our research was its mixed methods approach, which allowed for a deeper understanding of participants' experiences. Furthermore, the implementation facilitator and barriers found here offer a valuable learning opportunity as they likely apply to mentorship interventions in low-resource settings more generally.

\section{Conclusions}

In this paper, we report on the findings of a mixed-methods assessment of a multimodal mentorship intervention with surgical providers in Tanzania's Lake Zone to improve surgical quality. We found that a multimodal design, high-quality mentee-mentor relationships, and understanding of local context can optimize mentorship. The themes identified offer insights and lessons that can inform policy makers, intervention designers, and practitioners about successful implementation of surgical mentorship interventions. Future research should examine the cost-effectiveness of multimodal mentorship approaches and the contextual factors that are important to optimize surgical mentorship in African settings.

\section{Abbreviations}

SS2020: Safe Surgery 2020; SSC: Surgical Safety Checklist; Project ECHO: Project Extension for Community Healthcare Outcomes; OR: Operating Room; BMC: Bugando Medical Center.

\section{Supplementary Information}

The online version contains supplementary material available at https://doi. org/10.1186/s12960-021-00652-6.

\section{Additional file 1: Mentorship survey}

Additional file 2: Interview protocols for mentees, mentors and facility leaders and focus group discussion questions.

Additional file 3: Overall experience, overall satisfaction and support for continuation of the mentorship program.

Additional file 4: Changes made resulting from the mentorship program. Additional file 5: Areas of Mentorship desired and received by mentees. Additional file 6:Mentor characteristics desired by mentees and exhibited by mentors.

\section{Acknowledgements}

We would like to thank the Tanzania Ministry of Health, Community Development, Gender, Elderly and Children, the President's Office for Regional Administration and Local Government in Tanzania, and the Regional Medical Officers of Mara and Kagera regions for their support of the study. We would like to thank the members of the surgical teams, mentors, and facility leaders who gave so generously of their time for interviews. We would like to express our sincere appreciation to the leadership and mentors from Bugando Medical Center for their support of the Safe Surgery 2020 mentorship intervention. We would like to acknowledge and thank all organizations and individuals who participated in our consultation on the design of Safe Surgery 2020 as well as those organizations and individuals who have contributed to the larger Safe Surgery 2020 initiative including Assist International, Dalberg, Jhpiego, Life Box, Project ECHO, the Sterile Processing Education Charitable Trust, and the World Federation of Societies of Anaesthesiologists. We would like to offer our thanks to Sophie Blumert for her editing assistance. We are grateful to Dr. Christina Smiraglia and Professor Aisha K. Yousafzai for advice on the qualitative analysis.

\section{Authors' contributions}

SA conceptualized and designed the study, analyzed and interpreted the data, and drafted the manuscript. LT conceptualized the study, interpreted the data and drafted the manuscript. NAK conceptualized the study, and substantively revised the manuscript. CD substantively revised the manuscript. SJA analyzed and interpreted the quantitative data and substantively revised the manuscript. DB conceptualized the study and substantively revised the manuscript. DBA analyzed and interpreted the qualitative data and substantively revised the manuscript. MC substantively revised the manuscript. KC substantively revised the manuscript. EE substantively revised the manuscript. JE provided inputs on the context for the paper but had no role in analysis of the data; he substantively revised the manuscript. LF conceptualized the study, interpreted data, and substantively revised the manuscript. GCG provided inputs on the context for the paper but had no role in analysis of the data; he substantively revised the manuscript. AH conceptualized the study, interpreted the data and substantively revised the manuscript. YH substantively revised the manuscript. FK substantively revised the manuscript. BK substantively revised the manuscript. AK provided inputs on the context for the paper but had no role in analysis of the data; he substantively revised the manuscript. SK substantively revised the manuscript. IK assisted with translation for the interviews and substantively revised the manuscript. JGM conceptualized the study and substantively revised the manuscript. CR conceptualized the study and substantively revised the manuscript. SJS analyzed and interpreted the quantitative data and substantively revised the manuscript. MS designed the data collection tools, conducted focus groups and interviews, and substantively revised the study. JV conceptualized the study and substantively revised the manuscript. NZ conducted interviews, analyzed and interpreted the qualitative data, and substantively revised the manuscript. All authors read and approved the final manuscript.

\section{Funding}

This work was supported by the GE Foundation [Grant No. 28045607] and Elma Philanthropies [Grant No. 17-F0012]. GE Foundation and Elma Philanthropies as entities were not involved in the study design, data collection and analysis, the decision to submit the findings for publication, or the drafting of the manuscript. David Barash, as an individual, had a role in the preparation, review, and approval of the manuscript and is an author on this paper.

\section{Availability of data and materials}

The data set analyzed during the current study is available from the corresponding author on reasonable request. All requests for data must be approved by the Tanzania Ministry of Health, Community Development, Gender, Elderly and Children, in accordance with the data sharing agreement.

\section{Declarations}

Ethics approval and consent to participate

We received ethical approval from Harvard Medical School and Tanzania's National Medical Research Institute (Ref. NIMR/HQ/R.8a/Nol. IX/2515). The 
study was also approved and endorsed by regional medical officers and district medical officers the regions and districts in which the study was conducted. Hospital administrators and staff were also briefed on the study and offered support to the data collectors during the study period. Verbal consent was obtained in Swahili from interviewees at the beginning of interviews and focus groups.

\section{Consent for publication}

No individual person's data are presented in this article. In accordance with Tanzanian law, permission to publish this manuscript was sought and obtained from the National Institute for Medical Research.

\section{Competing interests}

The Program in Global Surgery and Social Change, Dalberg Advisors, Jhpiego, and Assist International receive funding from the GE Foundation for the Safe Surgery 2020 project. Shehnaz Alidina, Sakshie Alreja, Danta Bien-Aime, Monica Cainer, Kevin Charles, Caroline Damian, Edwin Ernest, Joachim Eyembe, Laura Fitzgerald, Geofrey C. Giiti, Augustino Hellar, Furaha Kahindo, Bernard Kenemo, Albert Kihunrwa, Steve Kisakye, Innocent Kissima, Cheri Reynolds, Meaghan Sydlowski, Leopold Tibyehabwa, John Varallo, and Noor Zanial had financial support from GE Foundation for the submitted work. David Barash is employed by GE Foundation, which funded this work. Edwin Ernest, Augustino Hellar, Laura Fitzgerald, Leopold Tibyehabwa, and John Varallo declare financial support from ELMA Philanthropies., Ntuli A. Kapologwe, Yahaya Hussein, Steven J. Staffa, and John Meara have nothing to declare. The authors have had no financial relationships with any organizations that might have an interest in the submitted work in the previous 3 years and no other relationships or activities that could appear to have influenced the submitted work.

\section{Author details}

${ }_{1}^{1}$ Program in Global Surgery and Social Change, Harvard Medical School, 641 Huntington Avenue, Boston, MA 02115, USA. ${ }^{2}$ Safe Surgery 2020 Project, Jhpiego, Dar es Salaam, Tanzania and Baltimore, MD, USA. ${ }^{3}$ GE Foundation, Boston, MA, USA. ${ }^{4}$ Assist International, Dar es Salaam, Tanzania and Ripon, CA, USA. ${ }^{5}$ Musoma Regional Hospital, Musoma, Tanzania. ${ }^{6}$ Department of Surgery and Department of Obstetrics and Gynaecology, Bugando Medical Center, Mwanza, Tanzania. ${ }^{7}$ Department of Health, Social Welfare, and Nutrition Service, President's Office - Regional Administration and Local Government, Dodoma, Tanzania. ${ }^{8}$ Nyakahanga Hospital, Karagwe, Tanzania. ${ }^{9}$ D-Implement, Dalberg Advisors, Dar es Salaam, Tanzania. ${ }^{10}$ Department of Plastic and Oral Surgery, Boston Children's Hospital, Boston, MA, USA. ${ }^{11}$ Department of Anesthesiology, Critical Care and Pain Medicine, Boston Children's Hospital, Boston, MA, USA. ${ }^{12}$ Department of Curative Services, Ministry of Health, Community Development, Gender, Elderly, and Children, Dodoma, Tanzania. ${ }^{13}$ Department of Paediatrics, University of Melbourne, Melbourne, Australia.

Received: 23 July 2021 Accepted: 1 September 2021

Published online: 23 September 2021

\section{References}

1. Meara JG, Leather AJ, Hagander L, Alkire BC, Alonso N, Ameh EA, et al. Global Surgery 2030: evidence and solutions for achieving health, welfare, and economic development. Lancet. 2015;386:569-624. https://doi. org/10.1016/S0140-6736(15)60160-X.

2. Biccard BM, Madiba TE, Kluyts HL, Munlemvo DM, Madzimbamuto FD, Basenero A, et al. Perioperative patient outcomes in the African Surgical Outcomes Study: a 7-day prospective observational cohort study. Lancet. 2018;391:1589-98. https://doi.org/10.1016/S0140-6736(18)30001-1.

3. Jumbam DT, Reddy CL, Makasa E, Boatin AA, Rogo K, Chu KM, et al. Investing in surgery: a value proposition for African leaders. Lancet. 2020;396:7-9. https://doi.org/10.1016/S0140-6736(20)30482-7.

4. WHO. Report on the burden of endemic health care-associated infection Worldwide Clean Care is Safer Care. Geneva: World Health Organization; 2011. p. 1-40.

5. Clack L, Willi U, Berenholtz S, Aiken AM, Allegranzi B, Sax H. Implementation of a surgical unit-based safety programme in African hospitals: a multicentre qualitative study. Antimicrob Resist Infect Control. 2019;8:91. https://doi.org/10.1186/s13756-019-0541-3.
6. https://blogs.worldbank.org/opendata/why-are-people-dying-followingsurgery-africa\#: : text=In\%20Africa\%2C\%20because\%20there\%20are ,occasionally\%20result\%20in\%20unnecessary\%20deaths Accessed March 102021

7. Kruk ME, Prescott MR, de Pinho H, Galea S. Are doctors and nurses associated with coverage of essential health services in developing countries? A cross-sectional study. Hum Resour Health. 2009;7:27. https://doi.org/10. 1186/1478-4491-7-27.

8. Alidina S, Menon G, Staffa SJ, Alreja S, Barash D, Barringer E, et al. Outcomes of a multicomponent safe surgery intervention in Tanzania's Lake Zone: a prospective, longitudinal study. Int J Qual Health Care. 2021;29:33. https://doi.org/10.1093/intqhe/mzab087.

9. Hellar A, Tibyehabwa L, Ernest E, Varallo J, Betram MM, Fitzgerald L, et al. A team-based approach to introduce and sustain the use of the WHO Surgical Safety Checklist in Tanzania. World J Surg. 2020;44:689-95. https:// doi.org/10.1007/s00268-019-05292-5.

10. Allegranzi B, Aiken AM, Zeynep Kubilay N, Nthumba P, Barasa J, Okumu $\mathrm{G}$, et al. A multimodal infection control and patient safety intervention to reduce surgical site infections in Africa: a multicentre, before-after, cohort study. Lancet Infect Dis. 2018;18:507-15. https://doi.org/10.1016/S14733099(18)30107-5.

11. Forrester JA, Starr N, Negussie T, Schaps D, Adem M, Alemu S, et al. Clean cut (adaptive, multimodal surgical infection prevention programme) for low-resource settings: a prospective quality improvement study. Br J Surg. 2020. https://doi.org/10.1002/bjs.11997.

12. Leslie HH, Gage A, Nsona H, Hirschhorn LR, Kruk ME. Training and supervision did not meaningfully improve quality of care for pregnant women or sick children in sub-Saharan Africa. Health Aff (Millwood). 2016;35:171624. https://doi.org/10.1377/hlthaff.2016.0261.

13. Ojemeni MT, Niles P, Mfaume S, Kapologwe NA, Deng L, Stafford R, et al. A case study on building capacity to improve clinical mentoring and maternal child health in rural Tanzania: the path to implementation. BMC Nurs. 2017;16:57. https://doi.org/10.1186/s12912-017-0252-0.

14. Arsenault C, Rowe SY, Ross-Degnan D, Peters DH, Roder-DeWan S, Kruk $M E$, et al. How does the effectiveness of strategies to improve healthcare provider practices in low-income and middle-income countries change after implementation? Secondary analysis of a systematic review. BMJ Qual Saf. 2021. https://doi.org/10.1136/bmjqs-2020-011717.

15. Rowe AK, Rowe SY, Peters DH, Holloway KA, Chalker J, Ross-Degnan D. Effectiveness of strategies to improve health-care provider practices in low-income and middle-income countries: a systematic review. Lancet Glob Health. 2018:6:e1163-75. https://doi.org/10.1016/S2214-109X(18) 30398-X.

16. Rowe AK, Rowe SY, Peters DH, Holloway KA, Ross-Degnan D. The effectiveness of training strategies to improve healthcare provider practices in low-income and middle-income countries. BMJ Glob Health. 2021;6:e003229. https://doi.org/10.1136/bmjgh-2020-003229.

17. USAID Mentoring for Human Capacity Development: Implementation principles and guidance 2018. https://www.mcsprogram.org/resource/ mentoring-human-capacity-development-implementation-principlesguidance/ Accessed April 52021

18. Schwerdtle P, Morphet J, Hall H. A scoping review of mentorship of health personnel to improve the quality of health care in low and middleincome countries. Glob Health. 2017;13:77. https://doi.org/10.1186/ s12992-017-0301-1.

19. Feyissa GT, Balabanova D, Woldie M. How effective are mentoring programs for improving health worker competence and institutional performance in Africa? A systematic review of quantitative evidence. J Multidiscip Healthc. 2019;12:989-1005. https://doi.org/10.2147/JMDH. S228951.

20. Mbonye MK, Burnett SM, Burua A, Colebunders R, Crozier I, Kinoti SN, et al. Effect of integrated capacity-building interventions on malaria case management by health professionals in Uganda: a mixed design study with pre/post and cluster randomized trial components. PLoS ONE. 2014;9: e84945. https://doi.org/10.1371/journal.pone.0084945.

21. Naikoba S, Senjovu KD, Mugabe P, McCarthy CF, Riley PL, Kadengye DT, et al. Improved HIV and TB knowledge and competence among midlevel providers in a cluster-randomized trial of one-on-one mentorship for task shifting. J Acquir Immune Defic Syndr. 2017;75:e120-7. https:// doi.org/10.1097/QAl.0000000000001378. 
22. Weaver MR, Burnett SM, Crozier I, Kinoti SN, Kirunda I, Mbonye MK, Naikoba S, Ronald A, Rubashembusya T, Zawedde S, Willis KS. Improving facility performance in infectious disease care in Uganda: a mixed design study with pre/post and cluster randomized trial components. PLOS ONE. 2014;9: e103017. https://doi.org/10.1371/journal.pone.0103017.

23. Magge H, Anatole M, Cyamatare FR, Mezzacappa C, Nkikabahizi F, Niyonzima S, et al. Mentoring and quality improvement strengthen integrated management of childhood illness implementation in rural Rwanda. Arch Dis Child. 2015;100:565-70. https://doi.org/10.1136/archdischi ld-2013-305863.

24. Manzi A, Nyirazinyoye L, Ntaganira J, Magge H, Bigirimana E, Mukanzabikeshimana $L$, et al. Beyond coverage: improving the quality of antenatal care delivery through integrated mentorship and quality improvement at health centers in rural Rwanda. BMC Health Serv Res. 2018;18:136. https:// doi.org/10.1186/s12913-018-2939-7.

25. Nkwawir SC, Batumani NN, Maruta T, Awasom CN. From grass to grace: How SLMTA revolutionised the Bamenda Regional Hospital Laboratory in Cameroon. Afr J Lab Med. 2014;3:203. https://doi.org/10.4102/ajlm.v3i2. 203.

26. Nzombe P, Luman ET, Shumba E, Mangwanya D, Simbi R, Kilmarx PH, et al. Maximising mentorship: variations in laboratory mentorship models implemented in Zimbabwe. Afr J Lab Med. 2014;3:241. https://doi.org/10. 4102/ajlm.v3i2.241.

27. Alidina S, Kuchukhidze S, Menon G, Citron I, Lama TN, Meara J, et al. Effectiveness of a multicomponent safe surgery intervention on improving surgical quality in Tanzania's Lake Zone: protocol for a quasi-experimental study. BMJ Open. 2019;9(10): e031800. https://doi.org/10.1136/bmjop en-2019-031800.

28. Pittalis C, Brugha R, Crispino G, Bijlmakers L, Mwapasa G, Lavy C, et al. Evaluation of a surgical supervision model in three African countriesprotocol for a prospective mixed-methods controlled pilot trial. Pilot Feasibility Study. 2019;5:25. https://doi.org/10.1186/s40814-019-0409-6.

29. Entezami P, Franzblau LE, Chung KC. Mentorship in surgical training: a systematic review. Hand (N Y). 2012;7:30-6. https://doi.org/10.1007/ s11552-011-9379-8.

30. Asiedu A, Nelson AR, Gomez PP, Tappis H, Effah F, Allen C. "It builds your confidence... you've done well": Healthcare workers' experiences of participating in a low-dose, high-frequency training to improve newborn survival on the day of birth in Ghana. Gates Open Res. 2019;3:1470. https://doi.org/10.12688/gatesopenres.12936.1.

31. Willcox M, Harrison H, Asiedu A, Nelson A, Gomez P, LeFevre A. Incremental cost and cost-effectiveness of low-dose, high-frequency training in basic emergency obstetric and newborn care as compared to status quo: part of a cluster-randomized training intervention evaluation in Ghana. Global Health. 2017;13:88. https://doi.org/10.1186/s12992-017-0313-X.

32. El-Gabri D, McDow AD, Quamme SP, Hooper-Lane C, Greenberg CC, Long $\mathrm{KL}$. Surgical coaching for advancement of global surgical skills and capacity: a systematic review. J Surg Res. 2020;246:499-505. https://doi.org/10. 1016/j.jss.2019.09.039.

33. Alidina S, Chatterjee P, Zanial N, Alreja SS, Balira R, Barash D, et al. Improving surgical quality in low-income and middle-income countries: why do some health facilities perform better than others? BMJ Qual Saf. 2021. https://doi.org/10.1136/bmjqs-2020-011795.

34. Alidina S, Zanial N, Meara JG, Barash D, Buberwa L, Chirangi B, et al. Applying the exploration, preparation, implementation, sustainment (EPIS) framework to safe surgery 2020 implementation in Tanzania's Lake Zone. J Am Coll Surg. 2020. https://doi.org/10.1016/j.jamcollsurg.2021.04.006.

35. Wurdeman T, Strader C, Alidina S, Barash D, Citron I, Kapologwe N, et al. In-hospital postoperative mortality rates for selected procedures in Tanzania's Lake Zone. World J Surg. 2021;45:41-9. https://doi.org/10.1007/ s00268-020-05802-w.

36. Curry LA, Krumholz HM, O'Cathain A, Plano Clark VL, Cherlin E, Bradley EH. Mixed methods in biomedical and health services research. Circ Cardiovasc Qual Outcomes. 2013;6:119-23. https://doi.org/10.1161/CIRCO UTCOMES.112.967885

37. Curry L, Nunez-Smith M. Mixed methods in health sciences research: a practical primer. London: Sage Publications; 2014.

38. Klassen AC, Creswell J, Plano Clark VL, Smith KC, Meissner HI. Best practices in mixed methods for quality of life research. Qual Life Res. 2012;21:377-80. https://doi.org/10.1007/s11136-012-0122-x.
39. English M, Schellenberg J, Todd J. Assessing health system interventions: key points when considering the value of randomization. Bull World Health Organ. 2011;89:907-12. https://doi.org/10.2471/BLT.11.089524.

40. Neubauer BE, Witkop CT, Varpio L. How phenomenology can help us learn from the experiences of others. Perspect Med Educ. 2019;8:90-7. https://doi.org/10.1007/s40037-019-0509-2.

41. Curry LA, Nembhard IM, Bradley EH. Qualitative and mixed methods provide unique contributions to outcomes research. Circulation. 2009;119:1442-52. https://doi.org/10.1161/CIRCULATIONAHA.107. 742775.

42. Tong A, Sainsbury P, Craig J. Consolidated criteria for reporting qualitative research (COREQ): a 32-item checklist for interviews and focus groups. Int J Qual Health Care. 2007;19:349-57. https://doi.org/10.1093/intqhc/ mzm042.

43. The National Road Map Strategic Plan to Improve Reproductive, Maternal Newborn, Child \& Adolescent Health in Tanzania (2016-2020) One Plan II (2016). United Republic of Tanzania.

44. DiMeo A, Lama T, Sydlowski M, Barash D, Maina E, Menon G, et al. A mixed-methods assessment of surgical capacity in Tanzania's Lake Zone. East Central Afr J Surg. 2021;26:6-14.

45. Dhanasekaran K, Babu R, Kumar V, Mehrotra R, Hariprasad R. Capacity building of gynecologists in cancer screening through hybrid training approach. J Cancer Educ. 2020;35(6):1243-9. https://doi.org/10.1007/ s13187-019-01589-0.

46. Hostutler CA, Valleru J, Maciejewski HM, Hess A, Gleeson SP, Ramtekkar UP. Improving pediatrician's behavioral health competencies through the project ECHO Teleconsultation Model. Clin Pediatr (Phila). 2020;59:1049_ 57. https://doi.org/10.1177/0009922820927018.

47. Zhou C, Crawford A, Serhal E, Kurdyak P, Sockalingam S. The impact of project ECHO on participant and patient outcomes: a systematic review. Acad Med. 2016:91:1439-61. https://doi.org/10.1097/ACM.0000000000 001328.

48. Bikinesi L, O'Bryan G, Roscoe C, Mekonen T, Shoopala N, Mengistu AT, et al. Implementation and evaluation of a Project ECHO telementoring program for the Namibian HIV workforce. Hum Resour Health. 2020;18:61. https://doi.org/10.1186/s12960-020-00503-w.

49. Kawasaki S, Francis E, Mills S, Buchberger G, Hogentogler R, Kraschnewski J. Multi-model implementation of evidence-based care in the treatment of opioid use disorder in Pennsylvania. J Subst Abuse Treat. 2019;106:5864. https://doi.org/10.1016/j.jsat.2019.08.016.

50. Strauss A, Corbin JM. Grounded theory in practice. Thousand Oaks: Sage Publications; 1997.

51. Saunders B, Sim J, Kingstone T, Baker S, Waterfield J, Bartlam B, et al. Saturation in qualitative research: exploring its conceptualization and operationalization. Qual Quant. 2018;52:1893-907. https://doi.org/10. 1007/s11135-017-0574-8.

52. Morse JM. The significance of saturation. Qual Health Res. 1995;5:147-9.

53. McHugh ML. Interrater reliability: the kappa statistic. Biochem Med (Zagreb). 2012;22:276-82

54. Judson K, Courtright P, Ravilla T, Khanna R, Bassett K. Impact of systematic capacity building on cataract surgical service development in 25 hospitals. BMC Ophthalmol. 2017;17:96. https://doi.org/10.1186/ s12886-017-0492-5.

55. Manzi A, Hirschhorn LR, Sherr K, Chirwa C, Baynes C, Awoonor-Williams $J K$, et al. Mentorship and coaching to support strengthening healthcare systems: lessons learned across the five Population Health Implementation and Training partnership projects in sub-Saharan Africa. BMC Health Serv Res. 2017;17(Suppl 3):831. https://doi.org/10.1186/ s12913-017-2656-7.

56. Belcher HME, Piggott DA, Sanders RA, Trent M. Research accountability groups and mentoring minutes: The $\mathrm{M}^{3}$ approach to promote public health infectious diseases research for diverse graduate students. Am J Orthopsychiatry. 2019;89:390-9. https://doi.org/10.1037/ort0000413.

57. Di Frances CD, Childs E, Fetterman JL, Villanti AC, Stanton CA, Russo AR, et al. Implementing and evaluating a mentor training to improve support for early-career scholars in tobacco regulatory science. Nicotine Tob Res. 2020;22:1041-5. https://doi.org/10.1093/ntr/ntz083.

58. Douglass K, Williams A. Faculty development program for emergency medicine physicians in India: a pilot program. AEM Educ Train. 2018;3(3):33-8. https://doi.org/10.1002/aet2.10125. 
59. Weber-Main AM, Shanedling J, Kaizer AM, Connett J, Lamere M, ElFakahany EE. A randomized controlled pilot study of the University of Minnesota mentoring excellence training academy: a hybrid learning approach to research mentor training. J Clin Transl Sci. 2019;18(3):152-64. https://doi.org/10.1017/cts.2019.368.

60. van der Windt M, van der Kleij RM, Snoek KM, Willemsen SP, Dykgraaf RHM, Laven JSE, et al. Impact of a blended periconception lifestyle care approach on lifestyle behaviors: before-and-after study. J Med Internet Res. 2020;22: e19378. https://doi.org/10.2196/19378.

61. World Health Organization. Bridging the "Know-Do" Gap: Meeting on Knowledge Translation in Global Health. Geneva, Switzerland. https:// www.measureevaluation.org/resources/training/capacity-building-resou rces/high-impact-research-training-curricula/bridging-the-know-do-gap. pdf. Accessed Jan 21, 2021

62. Hull L, Athanasiou T, Russ S. Implementation science: a neglected opportunity to accelerate improvements in the safety and quality of surgical care. Ann Surg. 2017;265:1104-12. https://doi.org/10.1097/SLA.00000 00000002013.

63. Pfeffer J, Sutton RI. The knowing-doing gap: How smart companies turn knowledge into action. 1st ed. Boston: Harvard Business School Press; 2000
64. Klein KJ, Sorra JS. The challenge of innovation implementation. Acad Manage Rev. 1996;21:1055-80. https://doi.org/10.2307/259164.

65. Bradley EH, Webster TR, Baker D, et al. Translating research into practice: speeding the adoption of innovative health care programs. Issue Brief (Commonw Fund). 2004;724:1-12.

66. Singer SJ, Hayes JE, Gray GC, Kiang MV. Making time for learning-oriented leadership in multidisciplinary hospital management groups. Health Care Manage Rev. 2015;40:300-12. https://doi.org/10.1097/HMR.0000000000 000037.

67. Aveling E-L, McCulloch P, Dixon-Woods M. A qualitative study comparing experiences of the surgical safety checklist in hospitals in high-income and low-income countries. BMJ Open. 2013;3: e003039. https://doi.org/ 10.1136/bmjopen-2013-003039.

68. Bergen N, Labonté R. "Everything is perfect, and we have no problems": detecting and limiting social desirability bias in qualitative research. Qual Health Res. 2020;30:783-92. https://doi.org/10.1177/1049732319889354.

\section{Publisher's Note}

Springer Nature remains neutral with regard to jurisdictional claims in published maps and institutional affiliations.
Ready to submit your research? Choose BMC and benefit from:

- fast, convenient online submission

- thorough peer review by experienced researchers in your field

- rapid publication on acceptance

- support for research data, including large and complex data types

- gold Open Access which fosters wider collaboration and increased citations

- maximum visibility for your research: over 100M website views per year

At BMC, research is always in progress.

Learn more biomedcentral.com/submissions 